\title{
Evidence for Segregated and Integrative Connectivity Patterns in the Human Basal Ganglia
}

\author{
Bogdan Draganski, ${ }^{1 \star}$ Ferath Kherif, ${ }^{1 \star}$ Stefan Klöppel, ${ }^{1,3,4}$ Philip A. Cook, ${ }^{5}$ Daniel C. Alexander, ${ }^{2}$ Geoff J. M. Parker, ${ }^{6}$ \\ Ralf Deichmann, ${ }^{1,7}$ John Ashburner, ${ }^{1}$ and Richard S. J. Frackowiak ${ }^{1,8,9}$ \\ ${ }^{1}$ Wellcome Trust Centre for Neuroimaging, Institute of Neurology, UCL, London WC1N 3BG, United Kingdom, ${ }^{2}$ Center for Medical Image Computing and \\ Department of Computer Science, UCL, London WC1E 6BT, United Kingdom, ${ }^{3}$ Department of Neurology, Neurozentrum, and ${ }^{4}$ Department of Psychiatry, \\ University Clinic Freiburg, 79106 Freiburg, Germany, ${ }^{5}$ Department of Radiology, University of Pennsylvania, Philadelphia, Pennsylvania 19104, ${ }^{6}$ Imaging \\ Science and Biomedical Engineering, University of Manchester, Manchester M13 9PL, United Kingdom, ${ }^{7}$ Brain Imaging Center, University Hospital \\ Frankfurt, 60596 Frankfurt, Germany, ${ }^{8}$ Département d’Études Cognitives, Ecole Normale Supérieure, 75005 Paris, France, and ${ }^{9}$ Laboratory of \\ Neuroimaging, Instituto di Ricovero e Cura a Carattere Scientifico Santa Lucia, 00179 Rome, Italy
}

Detailed knowledge of the anatomy and connectivity pattern of cortico-basal ganglia circuits is essential to an understanding of abnormal cortical function and pathophysiology associated with a wide range of neurological and neuropsychiatric diseases. We aim to study the spatial extent and topography of human basal ganglia connectivity in vivo. Additionally, we explore at an anatomical level the hypothesis of coexistent segregated and integrative cortico-basal ganglia loops. We use probabilistic tractography on magnetic resonance diffusion weighted imaging data to segment basal ganglia and thalamus in 30 healthy subjects based on their cortical and subcortical projections. We introduce a novel method to define voxel-based connectivity profiles that allow representation of projections from a source to more than one target region. Using this method, we localize specific relay nuclei within predefined functional circuits. We find strong correlation between tractography-based basal ganglia parcellation and anatomical data from previously reported invasive tracing studies in nonhuman primates. Additionally, we show in vivo the anatomical basis of segregated loops and the extent of their overlap in prefrontal, premotor, and motor networks. Our findings in healthy humans support the notion that probabilistic diffusion tractography can be used to parcellate subcortical gray matter structures on the basis of their connectivity patterns. The coexistence of clearly segregated and also overlapping connections from cortical sites to basal ganglia subregions is a neuroanatomical correlate of both parallel and integrative networks within them. We believe that this method can be used to examine pathophysiological concepts in a number of basal gangliarelated disorders.

Key words: basal ganglia; thalamus; diffusion weighted imaging (DWI); white matter; connectivity; cortex

\section{Introduction}

Fundamental to an understanding of the pathophysiological basis of various neurological and neuropsychiatric disorders is a detailed knowledge of the anatomy of circuits engaging basal ganglia nuclei and thalamus. In this study, we investigate in vivo patterns of cortical and subcortical basal ganglia connectivity and describe in detail distinct prefrontal, premotor, and motor cortico-basal ganglia loops.

Given that the striatum is the main input nucleus for projections from specific remote cortical areas and the pallidum for

\footnotetext{
Received Feb. 5, 2008; revised May 21, 2008; accepted May 28, 2008.

This work was supported by Wellcome Trust Grant 075696/Z/04/Z (R.S.J.F., Sarah Tabrizi, J.A.). We thank all participants in our study and the radiographers at the Functional Imaging Laboratory for their assistance acquiring data. We also thank Mathias Pessiglione, Guillaume Flandin, and Tim Behrens for their inspiring ideas and valuable comments on our work, Jean Daunizeau for the three-dimension rendering tools, and Anka Draganski for editing the figures.

*B.D. and F.K. contributed equally to this work.

This article is freely available online through the J Neurosci Open Choice option.

Correspondence should be addressed to Dr. Bogdan Draganski, Wellcome Trust Centre for Neuroimaging, 12 Queen Square, London WC1N 3BG, UK. E-mail: b.draganski@fil.ion.ucl.ac.uk.

D0I:10.1523/JNEUROSCI.1486-08.2008

Copyright $\odot 2008$ Society for Neuroscience $\quad 0270-6474 / 08 / 287143-10 \$ 15.00 / 0$
}

channeling output via thalamic targets (for review, see Haber, 2003; Utter and Basso, 2008), it is clear that the basal ganglia must have a major influence on sensorimotor, limbic, and cognitive information processing (Middleton and Strick, 2000, 2002). Presently, reward and reinforcement are assigned to ventral striatum (Schultz et al., 1997; Pessiglione et al., 2006), the central parts of the basal ganglia play a role in cognitive functions (Levy et al., 1997; Jog et al., 1999), and the dorsolateral putamen is known to be responsible for motor control (Alexander et al., 1986). Symptom distribution and patterns of dysfunction in distinct functional domains in various neurological and neuropsychiatric diseases are primarily confirmatory of this schema (McGuire et al., 1994; Pantelis et al., 1997; Utter and Basso, 2008).

Based on neurophysiological models and anatomical data, it is assumed that there are both parallel (Alexander et al., 1990; Middleton and Strick, 2002) and converging pathways in corticobasal ganglia loops (Takada et al., 1998a,b; Bar-Gad and Bergman, 2001; Haber, 2003). This parallel organization is not completely maintained through trans-thalamic circuits (Kolomiets et al., 2001; McFarland and Haber, 2002). It was therefore suggested that thalamic nuclei may play a dual role, beyond that of a simple 
relay function they may modulate information processing between segregated circuits (Castro-Alamancos and Connors, 1997).

Previous studies confirm the validity of probabilistic diffusion tractography for thalamic or striatal connectivity-based parcellation in vivo (Behrens et al., 2003; Johansen-Berg et al., 2005; Leh et al., 2007), and recent work has validated them against histological fiber tracing methods (Dyrby et al., 2007). Streamline tractography, despite insensitivity and hence lack of precision in deep gray matter structures with low fractional anisotropy (FA) values, has been used to demonstrate specific cortico-striatal connections in humans (Lehericy et al., 2004a,b). This study extends previous work in several ways. To our knowledge, no published work in the field of fiber tracking has yet considered using a voxel-based representation of anatomical connectivity to multiple target regions. Beyond the definition of connectivity patterns, our voxel connectivity profile (VCP) method allows detection of integrative basal ganglia or thalamic nuclear regions that project to segregated functionally related cortical areas.

The aim of this study was to examine in vivo basal ganglia and thalamic connectivity in humans. Our main goal was to provide evidence to support the hypothesis of coexisting segregated and integrative cortico-basal ganglia circuits from their connectivity patterns. Our study relies on fully automated algorithms, combining the advantage of intra-individual cortical and subcortical parcellation with a validated probabilistic fiber tracking method (Parker and Alexander, 2003, 2005; Newton et al., 2006; Klöppel et al., 2008).

\section{Materials and Methods}

Data acquisition. We acquired diffusion weighted images in 30 healthy subjects (13 females, 29 right-handed, mean age of 34 years, range of 23-58 years) on a 1.5 T Sonata Scanner (Siemens). Diffusion weighted imaging (DWI) was performed with an echo planar sequence comprising a double spin-echo module to reduce the effect of eddy currents (Reese et al., 2003). Data acquisition was cardiac gated to reduce motion artifacts from CSF pulsation (Wheeler-Kingshott et al., 2002). Each data volume consisted of 40 axial slices of $2.3 \mathrm{~mm}$ thickness with no interslice gaps and an acquisition matrix of $96 \times 96$ in a field of view $(\mathrm{FoV})$ of $220 \times 220$ $\mathrm{mm}$, resulting in $2.3 \times 2.3 \times 2.3 \mathrm{~mm}^{3}$ isotropic voxels [echo time (TE), $90 \mathrm{~ms}$; flip angle, $90^{\circ}$; fat saturation; bandwidth, $2003 \mathrm{~Hz} /$ pixel]. Each dataset consisted of 61 high-diffusion-weighted images $(b=1000$ $\mathrm{s} / \mathrm{mm}^{2}$ ), with diffusion gradients applied along 61 evenly distributed diffusion directions obtained from a previously reported optimization procedure (Jansons and Alexander, 2003) and seven additional images with minimal diffusion weighting $\left(b=100 \mathrm{~s} / \mathrm{mm}^{2}\right)$ and evenly distributed directions. The diffusion tensor was fitted using a standard linear least squares fit to the log measurements (Basser et al., 1994). Additionally, the fitting provides an effective $b=0$ image. From each subject, we also acquired high-resolution T1-weighted structural data using the modified driven equilibrium Fourier transform sequence [176 slices; 1 $\mathrm{mm}^{3}$ isotropic voxels; sagittal, phase encoding in anterior/posterior; FoV, $224 \times 256 \mathrm{~mm}$; matrix, $224 \times 256$; repetition time, $20.66 \mathrm{~ms}$; TE, $8.42 \mathrm{~ms}$; inversion time, $640 \mathrm{~ms}$; flip angle, 25 degrees; fat saturation; bandwidth, $178 \mathrm{~Hz} /$ pixel] (Deichmann, 2006).

Image analysis. We first describe the creation of seed and target maps in subject-specific native space using freely available software for automated segmentation and structure labeling (Desikan et al., 2006) (Freesurfer, available at http://surfer.nmr.mgh.harvard.edu). This is followed by a description of the fiber tracking algorithm, the generation of spatial transforms, and, finally, the statistical analysis.

Preparation of seed and target areas. To allow bias-free definition of seed and target areas unaffected by subjective judgments about anatomical correspondences, we performed automated cortical and subcortical segmentation and labeling in subject-specific native space.

As suggested previously (Behrens et al., 2003; Johansen-Berg et al.,
2005), we used all voxels in the basal ganglia structures and cortex to define seed points and target areas. An automated segmentation was performed on high-resolution T1-weighted scans in subject specific native space using the Freesurfer software. The structures caudate, putamen, pallidum, and thalamus were considered as separate seed areas. Forty-six automatically labeled cortical areas (23 per hemisphere) (Fig. $1 A$ ) (supplemental Fig. $1 B$, available at www.jneurosci.org as supplemental material) covering the whole cortex were defined as targets for probabilistic tracking. The hippocampus, fusiform gyrus, and substantia nigra were not included as target regions because of poor segmentation performance in these regions. For basal ganglia and thalamic subcortical connectivity analyses, caudate, putamen, pallidum, and thalamus served as both seed and target regions.

Probabilistic tracking. Probabilistic diffusion tractography was performed in subject-specific native space using the previously described probabilistic index of connectivity (PICo) algorithm (Parker and Alexander, 2003, 2005) implemented in the freely available Camino software package (http://www.cs.ucl.ac.uk/research/medic/camino/) (Cook et al., 2006). PICo involves repeated iterations of a streamline process using Monte Carlo methods to model inherent uncertainty in the orientation of the principal diffusion direction(s) defined for each voxel. First, we identified fiber crossings using the algorithm proposed by Alexander et al. (2002) and parameterized them with a mixture of two tensors (Tuch et al., 2002); in all other parenchymal voxels, a single tensor model was used. We then applied the method of Cook et al. (2004) to compute probability density functions of the fiber tract orientation estimated from the principal direction of diffusion tensor(s) in each voxel, under the assumption that dominant orientations derived from the diffusionweighted data correspond to the dominant orientations of fiber bundles. Stopping criteria prevented biologically implausible curvature of streamlines $\left(>80^{\circ}\right.$ on the scale of a single voxel) or attempts to transit voxels with FA value $<0.1$. Five thousand iterations were used to identify each connection route. Each seed point, its coordinate, and probability of connection to a given target area were used for additional analysis. PICo is derived as the proportion of the total number of samples from a seed voxel that reach a target area. Additionally, we calculated on a voxel-byvoxel basis, using Camino software, FA maps based on the three eigenvalues of each diffusion tensor (Pierpaoli and Basser, 1996; Pierpaoli et al., 1996).

Spatial transformation into standard Montreal Neurological Institute space. We created subject-by-subject individual maps of the basal ganglia ( 2 hemispheres $\times 4$ seed regions) containing voxel-based PICo values to either each of the 23 predefined anatomical regions in ipsilateral cortex or to each of the remaining ipsilateral basal ganglia nuclei. No threshold was applied at this stage. We then used spatial transformations to warp the voxel-based PICo values in seed areas from subject-specific native space to standard Montreal Neurological Institute (MNI) space. The individual transformation matrices were estimated after first coregistering T1weighted scans with their corresponding FA maps in native space. The coregistered structural images were segmented and transformed into standard MNI space using a unified segmentation implemented in SPM5 (Ashburner and Friston, 2005) running under Matlab7 (MathWorks). The parameters estimated by transforming T1-weighted images to a standardized anatomical template were applied to the individual seed PICo maps.

Voxel connectivity profiles-based segmentation. We calculated voxelbased average PICo maps for thalamic and basal ganglia seed structures to each cortical and subcortical target area using all the individual maps transformed to standard MNI space.

To minimize noise, we first thresholded the average PICo maps to include only those voxels with a probabilistic connectivity index of $>1 \%$. The thresholded average maps were then binarized, which resulted at the voxel level in binary strings of the same length representing connectivity patterns to all 23 cortical targets. Subsequently, each voxel (VCP) was labeled in ascending bit order on the basis of the Hamming distance between specific connectivity patterns (Fig. $1 A$ ). The Hamming distance represents an index of similarity between connectivity patterns so that all seed voxels with an identical VCP within a structure were assigned the same label (color), and similar VCPs received similar labels (color gradi- 


\section{A}

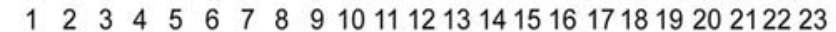

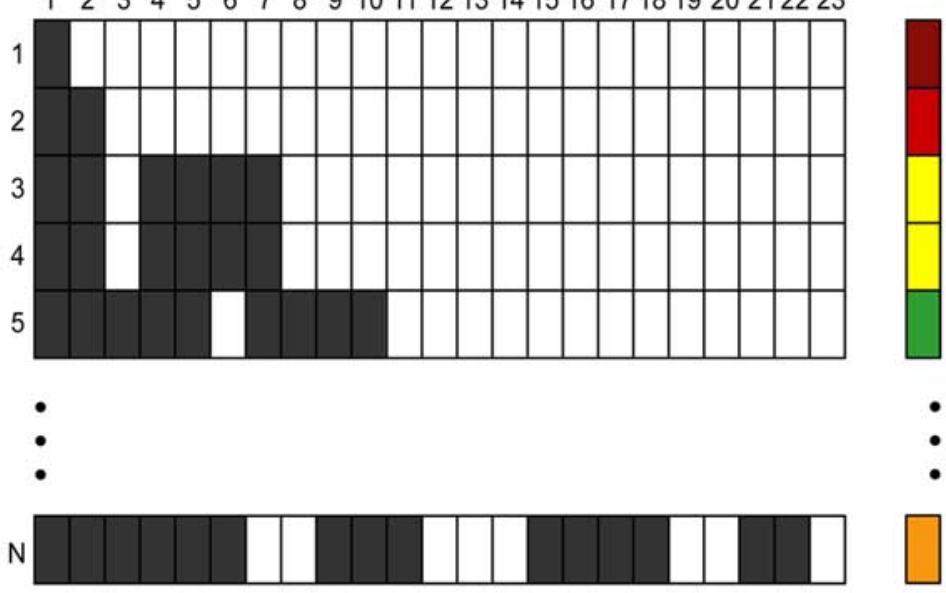

B
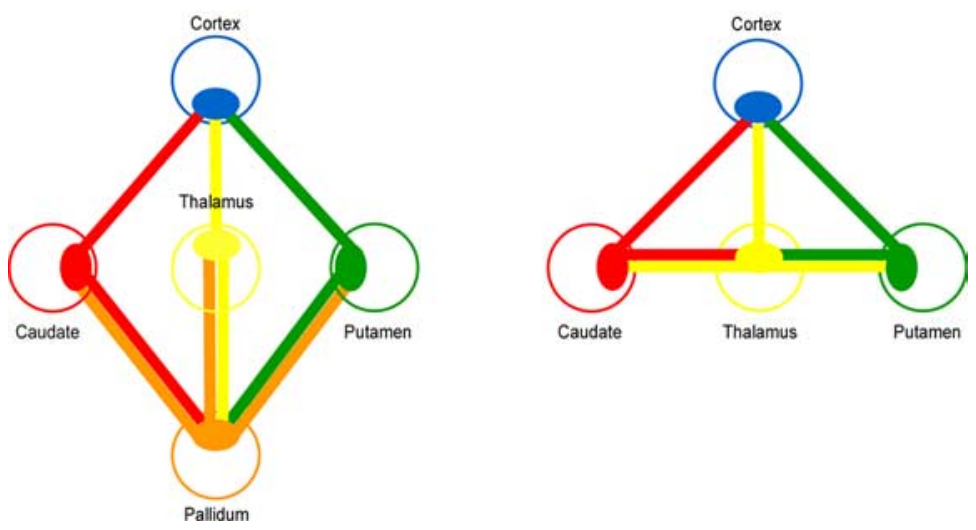

Figure 1. A, Example of voxel connectivity profiles (VCPs) of $n$ seed voxels (rows) projecting to 23 cortical target regions (columns). Filled and open squares correspond to binary code for existing versus not detectable connections above a probability threshold of 1\%. Each voxel is labeled according to its specific VCP in ascending bit order (voxels with identical VCPs receive the same color label). $\boldsymbol{B}$, Schematic representation of both basal ganglia models as intersection graphs. The subcortical seed regions are shown as colored open circles. Each relay nucleus (full ellipsoids) is projecting to all neighboring structures. The blue ellipsoid corresponds to a particular cortical target area (MPFC, OFC, DLPFC, premotor, or motor cortex).

ent). We used a hot-to-cold color gradient to represent the rostral-tocaudal distribution in both basal ganglia segmentation maps and VCP plots (see Figs. 2-4). Rather than attempt the description of all possible colors, of which there are many, we label those that cluster together and are distinguishable in figure planes (see Figs. 3, 4).

Additionally, we identified and labeled caudate, putamen, pallidum, and thalamus VCPs relative to each other (basal ganglia and thalamic targets) using the same analysis procedure and labeling convention (supplemental Fig. 3, available at www.jneurosci.org as supplemental material).

Basal ganglia relay structures in anatomically predefined circuits. In an additional analysis step based on either individual or averaged PICo maps, we localized the anatomical substrate of specific relay nuclei in predefined theoretically and neurophysiologically established corticobasal ganglia circuits. Using "intersection graphs" (McKee et al., 1999), we defined relays as those voxels that were connected to all associated structures in the predefined circuits (Fig. $1 B$ ). Two cortico-basal ganglia circuits each with five distinct cortical regions projecting to the following subcortical structures were explored: (1) cortico-striato-pallidothalamo-cortical loop ("long" loop) (Fig. 1B) (see Fig. 5): putamen, connections to both cortex (a, b, c, d, or e) and pallidum; caudate, con- nections to both cortex (a, b, c, d, or e) and pallidum; pallidum, connections to putamen, caudate, and thalamus; thalamus, connections to both pallidum and cortex (a, b, c, d, or e); (2) cortico-striato-thalamo-cortical loop ("short" loop) (Fig. 1B) (see Fig. 6): putamen, connections to both cortex (a, b, c, d, or e) and thalamus; caudate, connections to both cortex ( $\mathrm{a}, \mathrm{b}$, $c$, d, or e) and thalamus; thalamus, connections to putamen, caudate, and cortex (a, b, c, d, or e); a (brown), medial prefrontal cortex (MPFC); $b$ (red), orbitofrontal cortex (OFC); $c$ (yellow), dorsolateral prefrontal cortex (DLPFC); $d$ (green), premotor cortex, dorsal (PMd) and ventral $(\mathrm{PMv})$ premotor cortex; $e$ (blue), motor cortex (M1).

When tracking the short cortico-striatothalamo-cortical loop, we explicitly rule out pallidal connections concentrating only on those striatal voxel clusters in putamen and caudate that are connected exclusively with both thalamus and a cortical area. The same applies to the thalamus: only thalamic voxel clusters that are simultaneously connected to striatal structures (putamen and caudate) and a cortical area are identified.

For the prefrontal cortex, we used the nomenclature of Petrides and Pandya (2002) and identified MPFC (areas 10, 32, anterior-most portions of 24), OFC (areas 11, 13, 14, and 47/ 12 ), and DLPFC (areas 9/46, 46), corresponding to the automatically labeled cortical regions derived by Freesurfer. The premotor areas were allocated to the caudal portions of the middle frontal gyrus; the motor cortex included the rostral and caudal extents of the central sulcus as well as the paracentral lobule (supplemental Fig. 2, available at www.jneurosci.org as supplemental material) (see Figs. 5, 6).

This analysis, in addition to being performed in the group averaged data, was also done at the individual level (native and MNI space).

Three-dimensional reconstruction. To examine in detail the overlap between distinct cortico-basal ganglia loops originating from either connected or not connected cortical areas, we created a three-dimensional (3D) reconstruction of the corresponding basal ganglia relays. We used rendering tools implemented in SPM5 and Matlab7. The 3D reconstructions were performed in MNI space based on the averaged data of all 30 subjects. For better visualization of overlapping relay clusters we adjusted the transparency parameters so that their presentation is in composite colors. Non-overlapping relays are shown in opaque (see Figs. 5, 6). The corresponding twodimensional representation of relays and originating pathways is included as supplemental material (supplemental Figs. 4-10, available at www.jneurosci.org as supplemental material).

Reproducibility and individual variability. For quality control, we looked for the presence or absence of whole cortico-subcortical circuits in both native and standardized space at the individual level. For these analyses, we accepted as whole circuit only loops in which all predefined relays were present. No parameters or thresholds were altered for this validation.

\section{Results}

Averaged and individual probabilistic diffusion tractography data of 30 healthy subjects were classified on a voxelwise basis in native (only for individual data) and standard MNI space (for both individual and averaged data) according to their VCPs. This 
procedure showed, in both hemispheres, nearly symmetrical clusters of similarly connected basal ganglia voxels with either cortical or remaining basal ganglia nuclear target areas. Given the inability of diffusion weighted magnetic resonance imaging (MRI) to determine the anatomical polarity of fiber tracts, we note that these results describe connections devoid of information about their orientation. We use theoretically and neurophysiologically established models of basal ganglia connectivity to demonstrate the anatomical validity of our results.

\section{Segmentation based on cortico-striatal} and cortico-pallidal connectivity

In the caudate, we detected 58 VCPs on the left and 41 on the right, 140 and 122 in pallidum, 272 and 261 in putamen, and 170 and 186 in thalamus, but the majority of voxels clustered together $\sim 10-15$ different patterns of connectivity (Fig. 2). Structures considered in the literature as ventral striatum show predominant connections to prefrontal cortical regions. MPFC projects to the medial caudate head and the medial and ventral putamen. OFC connections occupy the adjacent central parts of the ventral striatum. The central and caudal portions of striatum are connected preferentially with DLPFC, premotor, sensorimotor, and parietal areas. We note the specific motor cortex (M1) connections to the dorsolateral portion of putamen caudal to the anterior commissure (Figs. 3, $4 B-D$, 5, 6) (supplemental Figs. 4-10, available at www.jneurosci.org as supplemental material).

The pallidum showed analogous topography, corresponding to a rostrocaudal gradient of prefrontal connections in addition to projections to sensorimotor and parietal cortical areas (Figs. 3, 4).

\section{Segmentation based on}

\section{thalamocortical connectivity}

Thalamic parcellation demonstrated distinct regions connected to either a single cortical region or to a combination of areas. In the region corresponding to the medial dorsal nucleus (MD), we detected connectivity to MPFC and OFC. The thalamic ventral anterior (VA) nucleus showed predominant projections to DLPFC, the ventral lateral (VL) nucleus to premotor and motor areas, and the ventral posterior nucleus to somatosensory cortical regions (Figs. 4A, 5, 6) (supplemental Figs. 6, 10, available at www.jneurosci.org as supplemental material).

\section{Segmentation based on striato-pallidal and} thalamo-pallidal connectivity

We tracked the connections between different nuclei of the basal ganglia despite the fact that some subregions are divided
LEFT

RIGHT

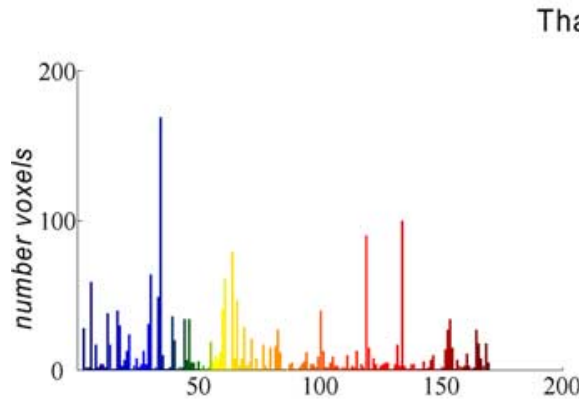

Thalamus

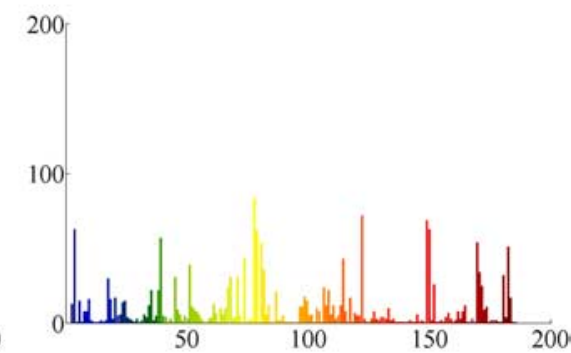

Caudate
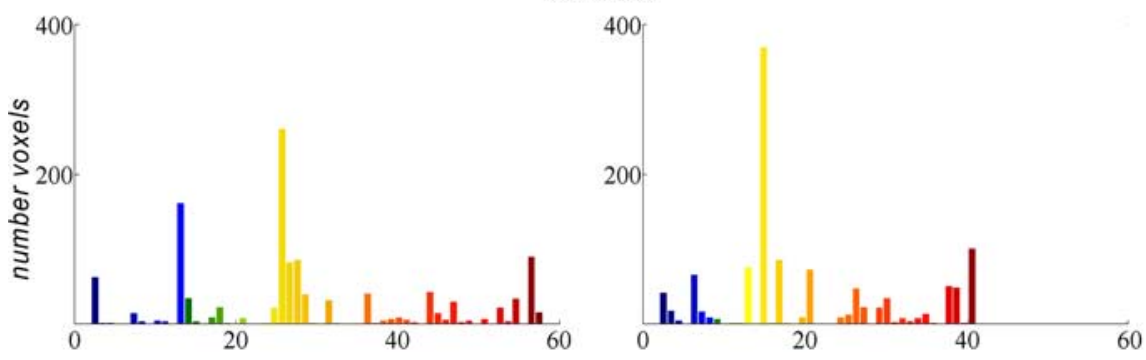

Putamen
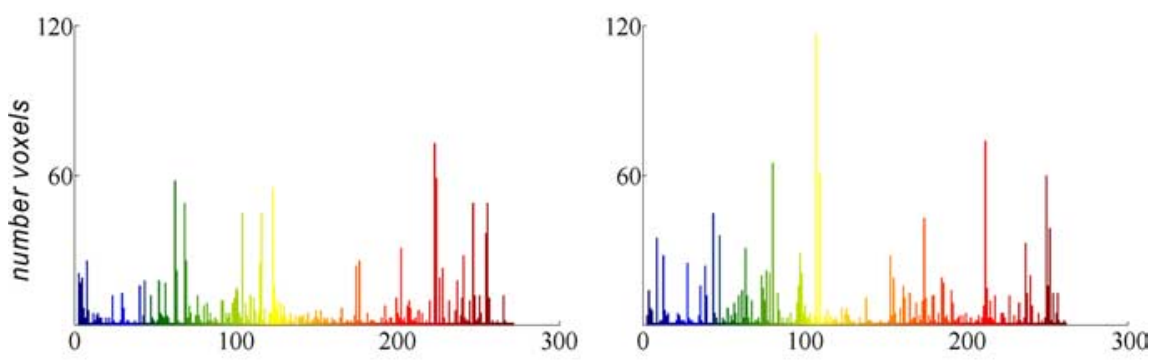

Pallidum

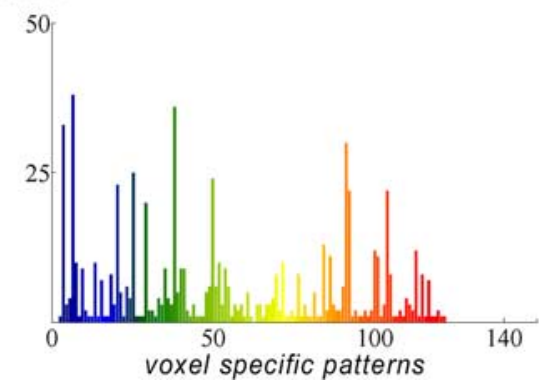

Figure 2. Plots demonstrating the number of voxels ( $y$-axis) for each specific voxel connectivity pattern ( $x$-axis) in the seed structures. Color pattern according rostral (hot colors) and caudal (cold colors) cortical distribution.

by distances smaller than our scan resolution of $2.3 \mathrm{~mm}$. For the basal ganglia-thalamic connections, we found eight VCPs. Caudate projections occupied mainly the dorsomedial pallidum, the putamen connected with the ventrolateral, and the thalamus with the ventromedial pallidum (supplemental Fig. $3 A-D$, available at www.jneurosci.org as supplemental material). tral striatal areas. Similarly, the lateral thalamic nuclei project to 

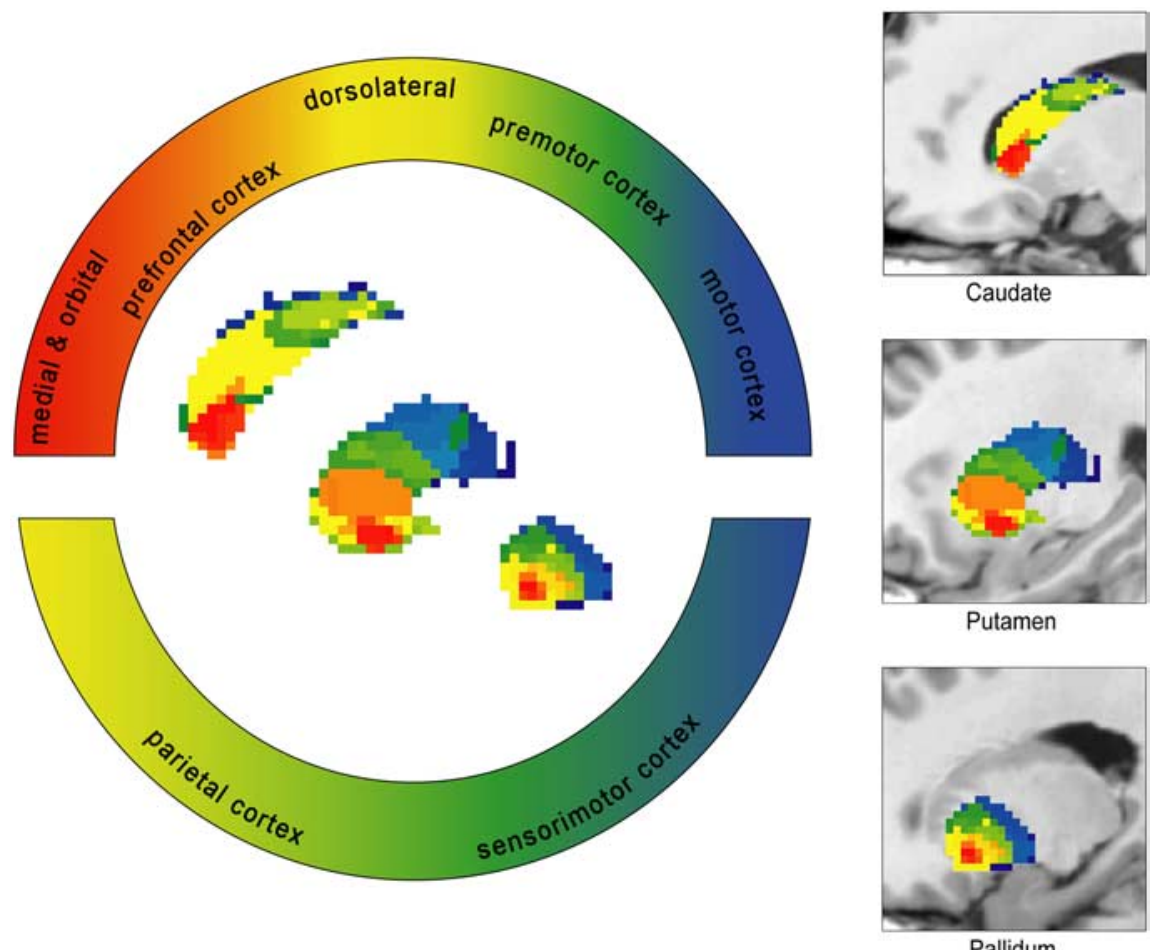

Figure 3. "Rostrocaudal gradient" of frontal cortical connectivity in caudate, putamen, and pallidum, sagittal view (after Haber, 2003). For better visualization, segmented basal ganglia nuclei are superimposed on a T1-weighted sagittal image. Areas of overlapping projections to both prefrontal and sensorimotor/parietal cortical regions are color coded using a different labeling convention (indicated in the bottom semicircular panel).

dorsal striatal areas (supplemental Fig. $3 A-D$, available at www. jneurosci.org as supplemental material).

\section{Cortico-basal ganglia circuits}

Cortico-striato-pallido-thalamo-cortical circuits

This section describes loops that traverse the pallidum to return to cortex. The pallidum is a common relay for all five corticosubcortical loops; its ventromedial subdivision connects with caudate, putamen, and thalamus.

MPFC (brown) and OFC (red). Symmetrical portions of the most ventral parts of caudate and putamen are connected with medial prefrontal, orbitofrontal cortical regions, and pallidum. The MD thalamic nucleus is occupied by projections to pallidum and MPFC/OFC. We observe in the MD nucleus an overlap between connections via pallidum and those arriving directly from MPFC/OFC via striatum alone (Figs. 5, 6) (supplemental Figs. 4-7, available at www.jneurosci.org as supplemental material).

DLPFC (yellow). DLPFC is connected diffusely to rostral and caudal components of both putamen and caudate, which are themselves connected to pallidum. These components contain both overlapping and non-overlapping patches with OFC/MPFC loop-associated connections. In thalamus, we detect relays connecting simultaneously to pallidum and DLPFC in a region localizable to rostral (VA nucleus) and medial dorsal thalamic (MD nucleus) subdivisions. The medial dorsal portions overlap to some extent with OFC loop-associated areas, whereas the ventral anterior parts do so with premotor cortex circuit relays (Fig. 5) (supplemental Figs. 4-7, available at www.jneurosci.org as supplemental material).

PMv and PMd (green). Dorsolateral putamen is connected to both premotor areas and pallidum, but we do not found any associ- ated relay structures in the caudate. The relays in thalamus projecting to pallidum and premotor areas localize to VA nucleus. Additionally, there is overlap with the putative thalamic VL nucleus as part of the motor loop (Fig. 5) (supplemental Figs. 4-7, available at www.jneurosci.org as supplemental material).

M1 (blue). The motor loop relays connecting with $\mathrm{M} 1$ and the pallidum occupy the dorsolateral putamen and dorsal caudate. In thalamus, we find an area corresponding to the VL nucleus, which projects to pallidum and motor cortex (Fig. 5) (supplemental Figs. 4-7, available at www.jneurosci.org as supplemental material).

Cortico-striato-thalamo-cortical circuits This section describes projections that bypass pallidum, connecting directly striatum and thalamus before returning to cortex.

MPFC (brown) and OFC (red). Areas of the striatum that connect with both MPFC and thalamus occupy the most ventral parts of putamen and caudate. Extending more centrally into the ventral striatum, we find subdivisions of putamen and caudate projecting to OFC and thalamus and also partially overlapping with an area that connects with MPFC and DLPFC. Thalamic connections with putamen, caudate, and MPFC/OFC occupy the MD nucleus in which both the short striato-thalamic and long (via pallidum) orbitofrontal loops show a high degree of overlap (Fig. 6) (supplemental Figs. 8-10, available at www. jneurosci.org as supplemental material).

DLPFC (yellow). Striatal connections to DLPFC and thalamus occupy the ventral striatum. We found graded overlap with MPFC/OFC loop-associated connections at this site. The thalamus has a similar distribution of connections with DLPFC, caudate, and putamen as in the loop that includes the pallidum but with extensions into thalamic VA and MD nuclei and dorsolateral subdivisions. There are similar areas of overlap involving short and long MPFC/OFC and DLPFC loops (Fig. 6) (supplemental Figs. 8-10, available at www.jneurosci.org as supplemental material).

PMv and PMd (green). We did not find caudate areas that project to thalamus and premotor areas. The central putamen connects to $\mathrm{PMv} / \mathrm{PMd}$ and thalamus, overlapping more ventrally with DLPFC and caudally with M1-associated connections. Thalamic regions with projections to both putamen and premotor areas are found in the region of the VA nucleus (Fig. 6) (supplemental Figs. 8-10, available at www.jneurosci.org as supplemental material).

M1 (blue). In the thalamo-striatal motor loop, we detect relay voxels in the dorsal putamen projecting to M1 and thalamus. There are no connections from the caudate to thalamus and premotor/motor loops. In the thalamus, the VL nucleus connects with both putamen and M1. These motor-associated areas overlap partially with the premotor loop (Fig. 6) (supplemental Figs. $8-10$, available at www.jneurosci.org as supplemental material). 
Reproducibility and

individual variability

Cortico-striato-pallido-thalamo-cortical circuits

Reproduction of the analysis with the same predefined cortical and subcortical areas at the individual level confirms the existence of the MPFC circuit bilaterally in 20 (23 in the left hemisphere, 20 in the right hemisphere) of 30 subjects in MNI space and in 24 subjects ( 28 in the left hemisphere, 24 in the right hemisphere) in native space. We detect whole OFC and DLPFC circuits in 30 of 30 subjects for both MNI and native space. The premotor cortex loop is present bilaterally in 26 subjects in MNI space and in 27 in native space. We find whole motor cortex circuits bilaterally in 20 (25 in the left hemisphere, 20 in the right hemisphere) of 30 subjects in MNI space and in 23 subjects ( 25 in the left hemisphere, 23 in the right hemisphere) in native space.

Cortico-striato-thalamo-cortical circuits At the individual level for the short thalamo-striatal loop, we detect MPFC circuits bilaterally in 20 (24 in the left hemisphere, 20 in the right hemisphere) of 30 subjects in MNI space and in 25 subjects (28 in the left hemisphere, 25 in the right hemisphere) in native space. We find the whole OFC circuit bilaterally in 29 of 30 subjects in MNI space and in 28 subjects (29 in the left hemisphere, 28 in the right hemisphere) in native space. The DLPFC circuit is detectable in all 30 subjects in both MNI and native space. Premotor cortical loops excluding the caudate are present bilaterally in 24 (25 in the left hemisphere, 24 in the right hemisphere) of 30 subjects in MNI space and in 24 subjects (26 in the left hemisphere, 25 in the right hemisphere) in native space. We confirm the existence of the motor cortex circuit excluding the caudate in all 30 subjects both for MNI and native space.

\section{Discussion}

Our study provides a detailed analysis of the cortical and subcortical connectivity patterns of human basal ganglia and thalamus captured using probabilistic diffusion tractography in vivo. Using the novel VCP method, we confirm the coexistence of both topographical segregation and a high degree of overlap between projections from specific frontal cortical areas. A voxel-based computation of intersections between VCPs demonstrates the spatial localization and extent of basal ganglia/thalamic subdivisions related to predefined functional networks, which therefore constitute anatomo-functionally defined relay "nuclei" of these systems. We achieve anatomical precision by using automated segmentation and labeling of individual brains to define seed and target areas. Our findings extend previously published work on thalamic and striatal anatomical connectivity to provide a reliable framework for studying potentially abnormal structural connec-
CORONAL

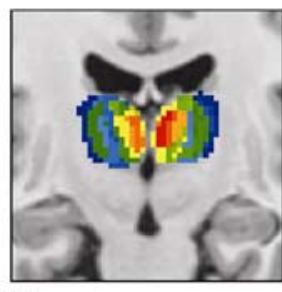

A. Thalamus

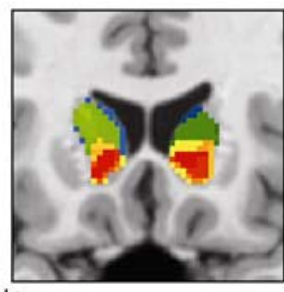

B. Caudate

Putamen
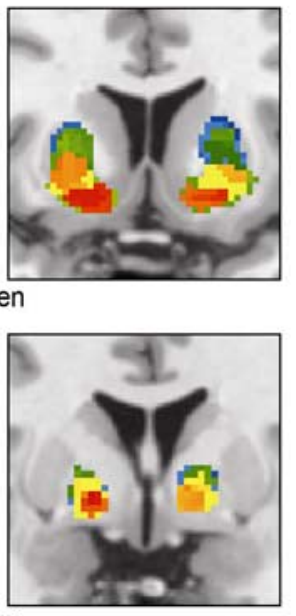

Pallidum
MPFC/OFC OFC/dACC OFC/DLPFC DLPFC/pre-motor cortex pre-motor/ motor cortex inferior parietal lobule, precuneus
Figure 4. Cortical connectivity pattern of the following: $\boldsymbol{A}$, thalamus; $\boldsymbol{B}$, caudate; $\boldsymbol{C}$, putamen; $\boldsymbol{D}$, pallidum (left column, axial view; right column, coronal view). The color labeling description of the voxel connectivity profile is restricted to the larger VCPs clusters. dACC, Anterior cingulate.

tivity patterns in various neurological and neuropsychiatric disorders.

Our motivation for exploring the specific spatial location of basal ganglia relays associated with predefined functional circuits was twofold. First, a demonstration of human basal ganglia and thalamic connectivity patterns proven anatomically in nonhuman primates would lend powerful face validity to our methods. Second, a direct comparison between the spatial extents of thalamic/basal ganglia projections to and from segregated functionally related cortical areas should provide reliable information about the existence of parallel and/or integrative pathways in and between these networks.

\section{Reward and motor system-related striatum}

Current thinking considers that, within the basal ganglia, the striatum is the main output structure, receiving afferents from specific cortical areas to project them on to the thalamus and brainstem for additional propagation via pallidum back to cortex (Haber, 2003). Evidence from previous studies confirms the central role of frontal cortical input to striatum in modulation of goal-directed behavior (Fuster, 2001). Rostral frontal regions, such as OFC and MPFC, are involved in processing different aspects of motivational context information for decision-making 


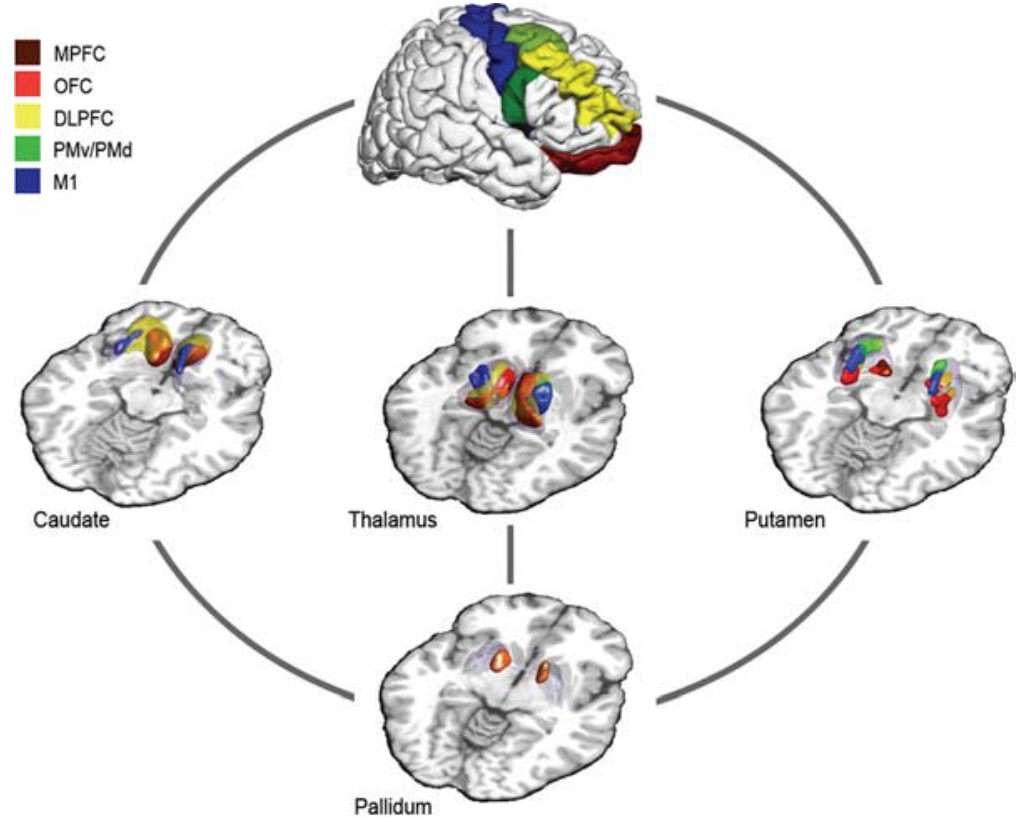

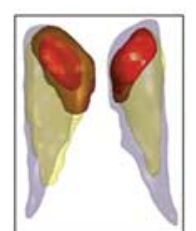

Caudate

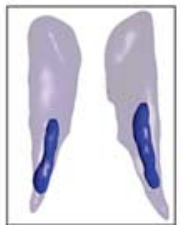

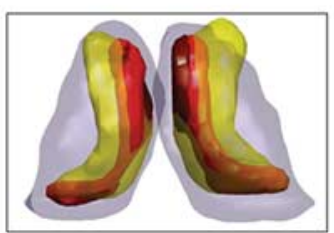

Thalamus

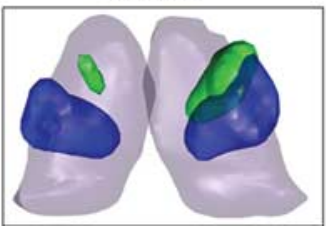

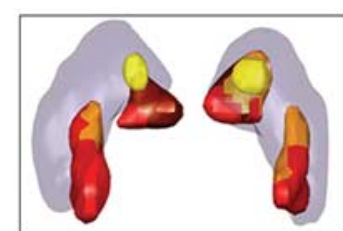

Putamen

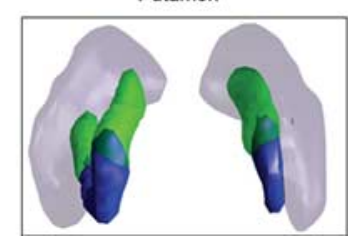

Figure 5. Long cortico-striato-pallido-thalamo-cortical circuit: intersection graph with cortical target areas: MPFC (brown), OFC (red), DLPFC (yellow), premotor areas (green), and motor cortex (blue). Caudate, putamen, pallidum, and thalamus are highlighted as transparent volumes, whereas the exact location of relays is highlighted in corresponding opaque colors. The overlap between relays from different circuits is represented as a color composite [e.g., orange, overlap between OFC (red) and DLPFC (yellow)]. Inset, Magnification of basal ganglia and thalamus, axial view.

(Elliott and Carson, 2000; Elliott et al., 2000; Fellows and Farah, 2005a,b), whereas DLPFC is important for cognitive contextual representation (Elliott et al., 2000). Premotor and motor areas, constituting the caudal frontal cortex, are responsible for action planning and execution. Consistent with a "functional gradient of inputs" to the striatum (Fuster, 2001; Haber, 2003), we demonstrate a similar, hierarchically organized, frontal cortical connectivity pattern (Fig. 3). OFC and MPFC connect with the ventral striatum, whereas caudal motor areas occupy exclusively dorsolateral portions, caudal to the anterior commissure. DLPFC projects extensively to the central striatum, thus bridging ventral and dorsal areas (Figs. 3, 5, 6).

The basal ganglia play a pivotal role in both selection of appropriate actions and their modulation by constantly changing internal and external contexts (Haber, 2003). Lesion studies, imaging, and neurophysiological experiments confirm the role of the ventral striatum in processing motivational context information (Schultz et al., 2003; Pessiglione et al., 2006), which contrasts with the involvement of dorsolateral striatum in motor control (Alexander et al., 1986; Taniwaki et al., 2003; Lehericy et al., 2006). This topographical segregation of frontal cortical path- ways is confirmed both by theoretical models of parallel cortico-basal ganglia pathways (Alexander et al., 1990) and by tracing studies in primates (Kunzle, 1978; Flaherty and Graybiel, 1994; Ferry et al., 2000; McFarland and Haber, 2000). However, accumulating knowledge about mechanisms of reward-based learning and goal-directed behavior supports the notion of integrative mechanisms linking information flow through otherwise segregated parallel pathways in cortico-basal ganglia networks (Bar-Gad and Bergman, 2001). Consistent with results from a recent invasive tracing study in primates (Haber et al., 2006), we demonstrate clearly defined striatal regions with overlap of connections with OFC, MPFC, or DLPFC (Figs. 5, 6). We interpret this finding as a morphological correlate of integration between functionally segregated reward-processing circuits. Previous work suggests a dopamine-dependent plasticity mechanism for reward-related learning in striatum that is important for decisionmaking (Tremblay et al., 1998; Schultz et al., 2003; Samejima et al., 2005; Pessiglione et al., 2006). Our results support this reinforcement learning model, providing insight into the anatomy of integrative striatal pathways enabling the transfer of reward representation from ventral striatum into optimal behavior output via dorsal striatum connections with DLPFC (Everitt and Robbins, 2005). We tested two models of connectivity, corticostriato-thalamo-pallido-cortical and cortico-striato-thalamo-cortical, and observe a similar pattern of segregated projection areas and regions of substantial overlap between specific, functionally specified loops (Figs. 5, 6).

We find that striatal motor projections occupy dorsolateral caudate and putamen, caudal to the anterior commissure (Figs. 5, 6) (supplemental Figs. 4, 5, 8, 9, available at www.jneurosci.org as supplemental material). Premotor projection areas in the putamen however, extend more rostrally, partially overlapping the motor areas, thus preserving the rostrocaudal functional gradient of inputs in striatum. Our findings are consistent with previous reports assigning an integrative area associated with sensorimotor control to the dorsal striatum (Alexander et al., 1990; Lehericy et al., 2006). Furthermore, the striatal connections with pallidum we find (Figs. $4 D, 5$ ) (supplemental Figs. 3D, 7) are in agreement with results from tracing studies showing connections between dorsolateral putamen and ventral pallidum (Francois et al., 1994; Parent et al., 2000; Saleem et al., 2002).

\section{Thalamic integrative nuclei}

Nonhuman primate tracer studies demonstrate major connections between thalamus and frontal cortex. Segregated circuit models suggest that the ventral thalamic nuclei are organized topographically in a hierarchical manner functioning to relay basal ganglia output pathways (Hoover and Strick, 1993; Matelli 
and Luppino, 1996). Our connectivity results support this view by demonstrating MD nuclear projections to MPFC and OFC. VA nucleus has predominant connections with motor planning-related premotor areas, whereas VL nucleus projects to the action execution associated cortical areas (Figs. 4, 5, 6) (supplemental Figs. 6, 10 , available at www.jneurosci.org as supplemental material).

By analogy to cortico-striatal pathways, there is however growing evidence to suggest a potential integrative role for thalamic nuclei in situations in which projections to functionally unrelated cortical areas converge (Sherman and Guillery, 1996; Darian-Smith et al., 1999). This idea gained credence from the finding that there are more cortico-thalamic than thalamo-cortical projections. Probability tracking based on DWI is unable to determine the polarity of connections so we are unable to prove the hypothesis. However, we do demonstrate substantial and coherent areas of overlap between areas connected to limbic cortical areas involved in decision-making (MPFC and OFC) and regions connected with working memory (DLPFC); the latter overlapping in turn with thalamic nuclei connected to action planning cortices (PMv, PMd). Connections to premotor areas in turn overlap with thalamic areas connected with executive motor cortex (Figs. 5, 6) (supplemental Figs. 6, 10, available at www.jneurosci.org as supplemental material).

We provide additional evidence to support the concept of integration in thalamic nuclei, based on overlap between specific cortico-subcortical loops, by demonstrating "direct" projections between thalamus and striatum, avoiding passage through pallidum (Fig. 6). Consistent with anatomical tracing studies in primates, we confirm a dual role for VA/VL thalamic nuclei that are connected with cortical, striatal, and pallidal regions. The connectivity pattern of the VA and VL nuclei supports the idea that they have a specific role integrating information from cortical motor areas with dorsal striatal output (Kultas-Ilinsky and Ilinsky, 1991; McFarland and Haber, 2002). Thus, we confirm at an anatomical level the notion that thalamic nuclei not only relay information through parallel pathways, but they also mediate integration between functionally specific cortical areas.

We report cortico-basal ganglia projections that replicate thalamic and striatal connectivity results of previous studies (Behrens et al., 2003; Lehericy et al., 2004a,b; Johansen-Berg et al., 2005; Leh et al., 2007). The main advance we contribute is the new VCP method because it permits the voxel-based representation of multiple targets rather than demonstrating connectivity overlap at the level of predefined regions (Behrens et al., 2003; Johansen-Berg et al., 2005). Unlike previous approaches based on a highest probability criterion that limit the description of connections from a seed voxel to a single target region, we identify specific VCPs that characterize projections from a seed voxel to single or multiple target areas (Fig. $1 A$ ). We find that, even when a large number of VCPs is found (e.g., 270 for cortico-putaminal connections), it is not randomly distributed but segregates into distinct and spatially continuous clusters with identical/similar VCP characteristics (Fig. 2). Confidence in our results is increased by the similar spatial distribution of connectivity patterns in left and right hemispheres, in which probabilistic tracking was performed independently. Additionally, we demonstrate high reproducibility rates, ranging between 67 and 100\% in individuals in both native and standardized MNI space.

In summary, we present a novel method for the analysis of probabilistic tractography data based on specific VCPs and provide a reliable framework for studying altered connectivity patterns in various neurological and neuropsychiatric disorders. However, obvious limitations of the imaging technique and preprocessing methods should be addressed. From an anatomical point of view, the main disadvantage of diffusion MRI is its inability to determine the polarity of connections. We performed probabilistic tractography in both directions between target and seed regions to provide evidence confirming the notion of coexistence of parallel and integrative pathways in anatomically and functionally specific cortico-subcortical loops. Because of the poor performance of automated segmentation in the region of the hippocampus, fusiform gyrus, and substantia nigra, we were unable to include these important structures in our analyses. Nevertheless, the anatomical precision obtained and the striking agreement with results from invasive tracing studies in nonhuman primates generates confidence in the validity of our inferences.

\section{References}

Alexander DC, Barker GJ, Arridge SR (2002) Detection and modeling of non-Gaussian apparent diffusion coefficient profiles in human brain data. Magn Reson Med 48:331-340.

Alexander GE, DeLong MR, Strick PL (1986) Parallel organization of func- 
tionally segregated circuits linking basal ganglia and cortex. Annu Rev Neurosci 9:357-381.

Alexander GE, Crutcher MD, DeLong MR (1990) Basal gangliathalamocortical circuits: parallel substrates for motor, oculomotor, "prefrontal" and "limbic" functions. Prog Brain Res 85:119-146.

Ashburner J, Friston KJ (2005) Unified segmentation. Neuroimage 26:839-851.

Bar-Gad I, Bergman H (2001) Stepping out of the box: information processing in the neural networks of the basal ganglia. Curr Opin Neurobiol 11:689-695.

Basser PJ, Mattiello J, LeBihan D (1994) MR diffusion tensor spectroscopy and imaging. Biophys J 66:259-267.

Behrens TE, Johansen-Berg H, Woolrich MW, Smith SM, Wheeler-Kingshott CA, Boulby PA, Barker GJ, Sillery EL, Sheehan K, Ciccarelli O, Thompson AJ, Brady JM, Matthews PM (2003) Non-invasive mapping of connections between human thalamus and cortex using diffusion imaging. Nat Neurosci 6:750-757.

Castro-Alamancos MA, Connors BW (1997) Thalamocortical synapses. Prog Neurobiol 51:581-606.

Cook PA, Alexander DC, Parker GJ (2004) Modelling noise-induced fibreorientation error in diffusion-tensor MRI. IEEE ISBI 1:332-336.

Cook PA, Bay Y, Nedjati-Gilani S, Seunarine KK, Hall MG, Parker GJ, Alexander DC (2006) Camino: open-source diffusion-MRI reconstruction and processing. Presented at the 14th Scientific Meeting of the International Society for Magnetic Resonance in Medicine, Seattle, WA, May.

Darian-Smith C, Tan A, Edwards S (1999) Comparing thalamocortical and corticothalamic microstructure and spatial reciprocity in the macaque ventral posterolateral nucleus (VPLc) and medial pulvinar. J Comp Neurol 410:211-234.

Deichmann R (2006) Fast structural brain imaging using an MDEFT sequence with a FLASH-EPI hybrid readout. Neuroimage 33:1066-1071.

Desikan RS, Ségonne F, Fischl B, Quinn BT, Dickerson BC, Blacker D, Buckner RL, Dale AM, Maguire RP, Hyman BT, Albert MS, Killiany RJ (2006) An automated labeling system for subdividing the human cerebral cortex on MRI scans into gyral based regions of interest. Neuroimage 31:968-980.

Dyrby TB, Søgaard LV, Parker GJ, Alexander DC, Lind NM, Baarée WF, Hay-Schmidt A, Eriksen N, Pakkenberg B, Paulson OB, Jelsing J (2007) Validation of in vitro probabilistic tractography. Neuroimage 37:1267-1277.

Elliott D, Carson RG (2000) Moving into the new millennium: some perspectives on the brain in action. Brain Cogn 42:153-156.

Elliott R, Friston KJ, Dolan RJ (2000) Dissociable neural responses in human reward systems. J Neurosci 20:6159-6165.

Everitt BJ, Robbins TW (2005) Neural systems of reinforcement for drug addiction: from actions to habits to compulsion. Nat Neurosci 8:1481-1489.

Fellows LK, Farah MJ (2005a) Dissociable elements of human foresight: a role for the ventromedial frontal lobes in framing the future, but not in discounting future rewards. Neuropsychologia 43:1214-1221.

Fellows LK, Farah MJ (2005b) Different underlying impairments in decision-making following ventromedial and dorsolateral frontal lobe damage in humans. Cereb Cortex 15:58-63.

Ferry AT, Ongür D, An X, Price JL (2000) Prefrontal cortical projections to the striatum in macaque monkeys: evidence for an organization related to prefrontal networks. J Comp Neurol 425:447-470.

Flaherty AW, Graybiel AM (1994) Input-output organization of the sensorimotor striatum in the squirrel monkey. J Neurosci 14:599-610.

François C, Yelnik J, Percheron G, Fénelon G (1994) Topographic distribution of the axonal endings from the sensorimotor and associative striatum in the macaque pallidum and substantia nigra. Exp Brain Res 102:305-318.

Fuster JM (2001) The prefrontal cortex-an update: time is of the essence. Neuron 30:319-333.

Haber SN (2003) The primate basal ganglia: parallel and integrative networks. J Chem Neuroanat 26:317-330.

Haber SN, Kim KS, Mailly P, Calzavara R (2006) Reward-related cortical inputs define a large striatal region in primates that interface with associative cortical connections, providing a substrate for incentive-based learning. J Neurosci 26:8368-8376.

Hoover JE, Strick PL (1993) Multiple output channels in the basal ganglia. Science 259:819-821.
Jansons KM, Alexander DC (2003) Persistent angular structure: new insights from diffusion MRI data. Dummy version. Inf Process Med Imaging 18:672-683.

Jog MS, Kubota Y, Connolly CI, Hillegaart V, Graybiel AM (1999) Building neural representations of habits. Science 286:1745-1749.

Johansen-Berg H, Behrens TE, Sillery E, Ciccarelli O, Thompson AJ, Smith SM, Matthews PM (2005) Functional-anatomical validation and individual variation of diffusion tractography-based segmentation of the human thalamus. Cereb Cortex 15:31-39.

Klöppel S, Draganski B, Golding CV, Chu C, Nagy Z, Cook PA, Hicks SL, Alexander DC, Parker GJ, Tabrizi SJ, Frackowiak RS (2008) White matter connections reflect changes in voluntary-guided saccades in presymptommatic Huntington's disease. Brain 131:196-204.

Kolomiets BP, Deniau JM, Mailly P, Ménétrey A, Glowinski J, Thierry AM (2001) Segregation and convergence of information flow through the cortico-subthalamic pathways. J Neurosci 21:5764-5772.

Kultas-Ilinsky K, Ilinsky IA (1991) Fine structure of the ventral lateral nucleus (VL) of the Macaca mulatta thalamus: cell types and synaptology. J Comp Neurol 314:319-349.

Künzle H (1978) An autoradiographic analysis of the efferent connections from premotor and adjacent prefrontal regions (areas 6 and 9) in Macaca fascicularis. Brain Behav Evol 15:185-234.

Leh SE, Ptito A, Chakravarty MM, Strafella AP (2007) Fronto-striatal connections in the human brain: a probabilistic diffusion tractography study. Neurosci Lett 419:113-118.

Lehéricy S, Ducros M, Krainik A, Francois C, Van de Moortele PF, Ugurbil K, Kim DS (2004a) 3-D diffusion tensor axonal tracking shows distinct SMA and pre-SMA projections to the human striatum. Cereb Cortex 14:1302-1309.

Lehéricy S, Ducros M, Van de Moortele PF, Francois C, Thivard L, Poupon C, Swindale N, Ugurbil K, Kim DS (2004b) Diffusion tensor fiber tracking shows distinct corticostriatal circuits in humans. Ann Neurol 55:522-529.

Lehéricy S, Bardinet E, Tremblay L, Van de Moortele PF, Pochon JB, Dormont D, Kim DS, Yelnik J, Ugurbil K (2006) Motor control in basal ganglia circuits using fMRI and brain atlas approaches. Cereb Cortex 16:149-161.

Levy R, Friedman HR, Davachi L, Goldman-Rakic PS (1997) Differential activation of the caudate nucleus in primates performing spatial and nonspatial working memory tasks. J Neurosci 17:3870-3882.

Matelli M, Luppino G (1996) Thalamic input to mesial and superior area 6 in the macaque monkey. J Comp Neurol 372:59-87.

McFarland NR, Haber SN (2000) Convergent inputs from thalamic motor nuclei and frontal cortical areas to the dorsal striatum in the primate. J Neurosci 20:3798-3813.

McFarland NR, Haber SN (2002) Thalamic relay nuclei of the basal ganglia form both reciprocal and nonreciprocal cortical connections, linking multiple frontal cortical areas. J Neurosci 22:8117-8132.

McGuire PK, Bench CJ, Frith CD, Marks IM, Frackowiak RS, Dolan RJ (1994) Functional anatomy of obsessive-compulsive phenomena. Br J Psychiatry 164:459-468.

McKee TA, Terry A, McMorris FR (1999) Topics in intersection graph theory. Philadelphia: Society for Industrial and Applied Mathematics.

Middleton FA, Strick PL (2000) Basal ganglia output and cognition: evidence from anatomical, behavioral, and clinical studies. Brain Cogn 42:183-200.

Middleton FA, Strick PL (2002) Basal-ganglia 'projections' to the prefrontal cortex of the primate. Cereb Cortex 12:926-935.

Newton JM, Ward NS, Parker GJ, Deichmann R, Alexander DC, Friston KJ, Frackowiak RS (2006) Non-invasive mapping of corticofugal fibres from multiple motor areas-relevance to stroke recovery. Brain 129:1844-1858.

Pantelis C, Barnes TR, Nelson HE, Tanner S, Weatherley L, Owen AM, Robbins TW (1997) Frontal-striatal cognitive deficits in patients with chronic schizophrenia. Brain 120:1823-1843.

Parent A, Sato F, Wu Y, Gauthier J, Lévesque M, Parent M (2000) Organization of the basal ganglia: the importance of axonal collateralization. Trends Neurosci 23:S20-S27.

Parker GJ, Alexander DC (2003) Probabilistic Monte Carlo based mapping of cerebral connections utilising whole-brain crossing fibre information. Inf Process Med Imaging 18:684-695.

Parker GJ, Alexander DC (2005) Probabilistic anatomical connectivity de- 
rived from the microscopic persistent angular structure of cerebral tissue. Philos Trans R Soc Lond B Biol Sci 360:893-902.

Pessiglione M, Seymour B, Flandin G, Dolan RJ, Frith CD (2006) Dopamine-dependent prediction errors underpin reward-seeking behaviour in humans. Nature 442:1042-1045.

Petrides M, Pandya DN (2002) Comparative cytoarchitectonic analysis of the human and the macaque ventrolateral prefrontal cortex and corticocortical connection patterns in the monkey. Eur J Neurosci 16:291-310.

Pierpaoli C, Basser PJ (1996) Toward a quantitative assessment of diffusion anisotropy. Magn Reson Med 36:893-906.

Pierpaoli C, Jezzard P, Basser PJ, Barnett A, Di Chiro G (1996) Diffusion tensor MR imaging of the human brain. Radiology 201:637-648.

Reese TG, Heid O, Weisskoff RM, Wedeen VJ (2003) Reduction of eddycurrent-induced distortion in diffusion MRI using a twice-refocused spin echo. Magn Reson Med 49:177-182.

Saleem KS, Pauls JM, Augath M, Trinath T, Prause BA, Hashikawa T, Logothetis NK (2002) Magnetic resonance imaging of neuronal connections in the macaque monkey. Neuron 34:685-700.

Samejima K, Ueda Y, Doya K, Kimura M (2005) Representation of actionspecific reward values in the striatum. Science 310:1337-1340.

Schultz W, Dayan P, Montague PR (1997) A neural substrate of prediction and reward. Science 275:1593-1599.

Schultz W, Tremblay L, Hollerman JR (2003) Changes in behavior-related neuronal activity in the striatum during learning. Trends Neurosci 26:321-328.
Sherman SM, Guillery RW (1996) Functional organization of thalamocortical relays. J Neurophysiol 76:1367-1395.

Takada M, Tokuno H, Nambu A, Inase M (1998a) Corticostriatal projections from the somatic motor areas of the frontal cortex in the macaque monkey: segregation versus overlap of input zones from the primary motor cortex, the supplementary motor area, and the premotor cortex. Exp Brain Res 120:114-128.

Takada M, Tokuno H, Nambu A, Inase M (1998b) Corticostriatal input zones from the supplementary motor area overlap those from the contrarather than ipsilateral primary motor cortex. Brain Res 791:335-340.

Taniwaki T, Okayama A, Yoshiura T, Nakamura Y, Goto Y, Kira J, Tobimatsu S (2003) Reappraisal of the motor role of basal ganglia: a functional magnetic resonance image study. J Neurosci 23:3432-3438.

Tremblay L, Hollerman JR, Schultz W (1998) Modifications of reward expectation-related neuronal activity during learning in primate striatum. J Neurophysiol 80:964-977.

Tuch DS, Reese TG, Wiegell MR, Makris N, Belliveau JW, Wedeen VJ (2002) High angular resolution diffusion imaging reveals intravoxel white matter fiber heterogeneity. Magn Reson Med 48:577-582.

Utter AA, Basso MA (2008) The basal ganglia: an overview of circuits and function. Neurosci Biobehav Rev 32:333-342.

Wheeler-Kingshott CA, Hickman SJ, Parker GJ, Ciccarelli O, Symms MR, Miller DH, Barker GJ (2002) Investigating cervical spinal cord structure using axial diffusion tensor imaging. Neuroimage 16:93-102. 